\title{
Application of multidimensional scaling method for analysis of EEG features in patients with dyscirculatory encephalopathy
}

\author{
Kaleriya Moroz ${ }^{1, *}$,Elena Kizhevatova $^{2}$, Vitaly Omelchenko ${ }^{1}$, Karina Karakhanyan $^{2}$, and \\ Natalia Korotkieva ${ }^{2}$ \\ ${ }^{1}$ Don State Technical University, Gagarina sq., 1, Rostov-on-Don, 344003, Russia \\ ${ }^{2}$ Rostov State Medical University, Nahichevansky av., 29, Rostov-on-Don, 344022, Russia
}

\begin{abstract}
The object of our work is to test the possibility of using the method of multidimensional scaling to identify the features of EEG in order to classify subjects in patients with dyscirculatory encephalopathy and healthy people by establishing differences in the graphs of the dynamics of the location of vectors in the multidimensional coordinate space. For healthy subjects, in almost all tests, there is a coincidence or close location of the spatial coordinates of the multidimensional scaling. For patients with dyscirculatory encephalopathy, there is no marked coincidence of coordinates; however, in some cases it is possible to group the data into local areas according to individual tests. The use of the method of multidimensional scaling in the diagnosis of dyscirculatory encephalopathy allows the analysis of the patient's condition, makes it possible to identify the degree of the disease
\end{abstract}

\section{Introduction}

The last 15 years have been characterized by the widespread introduction of new and significant improvement of already known functional diagnostic methods of brain research. Against this background, the method of assessing the functioning of the brain by its electrical activity - electroencephalography (EEG) - has not lost its importance. For over 80 years, EEG has been widely used in research of diseases of the nervous system, but the clinical significance of a number of phenomena detected by it continues to be the subject of debate due to the complexity of the EEG signal. For example, here are some papers that describe how EEG diagnostics can be used in cognitive impairment, in case Alzheimer's disease and other $[1,2]$. With the improvement of computer diagnostics software, along with the generally accepted mathematical methods of processing biosignals, the use of multidimensional statistical methods that allow obtaining sorted information from the total number of data sets is promising. For example, the literature describes methods for recognizing superimposed EEG rhythms with multidimensional decomposition [3], parameter investigation of topological data analysis for EEG signals [4], spectral analysis applications, connectome spectral analysis to track EEG task dynamics on a subsecond

*Corresponding author: leramoroz@mail.ru 
scale [5]. And also a description of the application of many other mathematical methods in EEG analysis.

The results of numerous studies clearly indicate that the bioelectric activity of the brain recorded with the help of a standard EEG is a derivative of cortical processes in which cognitive activity is reflected [6- 8]. Thus, it is quite relevant to study the bioelectric activity of the brain in patients with encephalopathies and subsequent cognitive impairment to identify the main parameters of EEG, which will be an additional criterion in the correct diagnosis.

Thus, the purpose of this work is to use the method of multidimensional scaling for EEG analysis, to demonstrate the use of information technologies to increase the informativeness of computer EEG analysis in assessing cognitive functions in patients with dyscirculatory encephalopathy.

\section{Materials and methods}

This study involved 60 people: patients with dyscirculatory encephalopathy and a control group (healthy individuals). In the group of patients with dyscirculatory encephalopathy there were 12 women and 18 men. The mean age of patients with dyscirculatory encephalopathy was $69.1 \pm 2.6(\mathrm{p} \geq 0.95)$ years. The control group consisted of healthy individuals 28-75 years old, the total number of which was 30 people (16 women and 14 men).

Recording of bioelectric activity of the brain (Background recording: relaxed state, eyes closed) was carried out on a 16-channel electroencephalograph "Encephalan-131-03".

Diagnosis of cognitive disorders was based on subjective complaints of the patient, a survey of their relatives, the assessment of neurological status, the results of psychological testing. Clinical assessment of patients was carried out with the help of neurologists at the Nervous Diseases and Neurosurgery Department. For clinical assessment of the psychological state of patients, the most common symptoms presented at the doctor's appointment were: mood decline, anxiety, general weakness, headache, relationship problems with others, inability to cope with the situation, violation of the waking-sleep regime, complaints about poor memory, decreased intellectual abilities.

The following tests were used as a screening study of cognitive impairment: "5 words"; Schulte test; hours drawing test; mini-study of the mental status of the subjects; Spielberger-Hanin reactive and personal anxiety test; Lüscher color test.

The task set earlier was solved by verbal tests: background registration of EEG with open and closed eyes ( 3 minutes) was carried out for a patient with a clinically identified syndrome of moderate cognitive disorders, then two verbal tests "Words" and "Plants" were offered with open eyes and EEG was registered. The performance of these tests causes the activation of the most suffering from chronic cerebral ischemia intellectual functions (attention, memory, speech activity, thinking, etc.). Bioelectric activity of the most involved areas of the brain (frontal and temporal leads), as well as neighboring areas that provide integrative activity, is a reflection of cognitive processes, including pathological ones.

After receiving the data of bioelectric signals, they were processed using MS Excel 2007 (We built spectra of patients and healthy people on the same graph, data preparation for Statistica), Statistica 6.0 (discriminant analysis and multidimensional scaling) and specialized software "Encephalan" version 2014.

Multidimensional scaling refers to classification analysis methods and to methods of simplifying the geometric structure of data. Multivariate scaling is often seen as an alternative to factor analysis, in which a reduction in the number of variables is achieved by isolating latent factors that explain the relationships between the observed variables. The purpose of multivariate scaling is to find and interpret latent variables that make it possible 
to explain similarities between objects. Multidimensional scaling is not just a specific procedure, but also rather a way to measure objects in the most efficient way, approximately preserving the observed distances between them. Multidimensional scaling is a popular method for statistical analysis of complex signals. many scientists in different countries use it to study large data sets of different types [9- 12].

\section{Results}

When comparing all EEG leads on the tests "Eyes open", "Eyes closed", "Plants", "Counting" in healthy subjects, the coincidence of spatial coordinates of multidimensional scaling is observed in almost all tests (figure 1). In some cases, there is a difference between the "Counting" test (figure 2), or the "Plants" test, and the rest of the tests (figure $3)$.

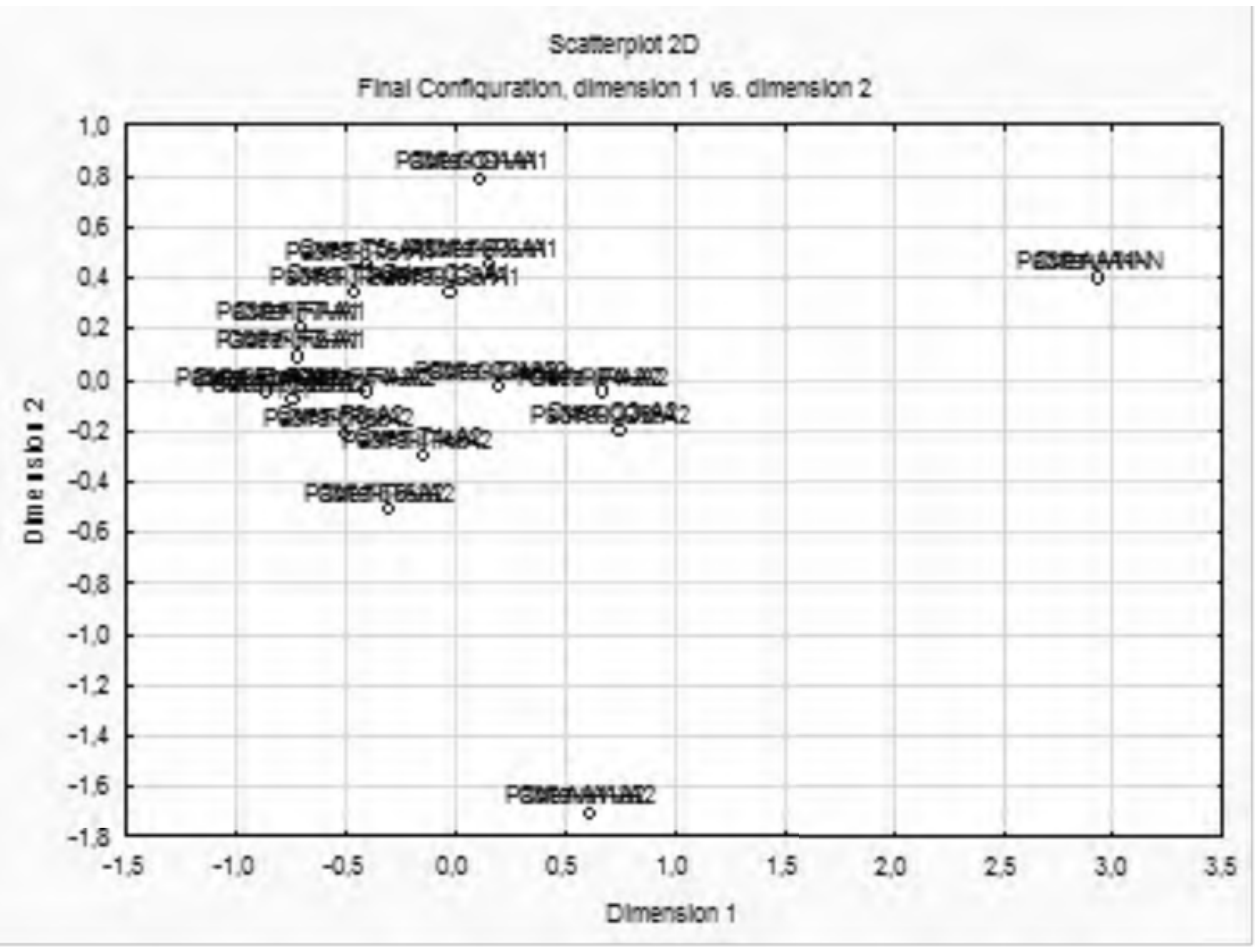

Fig. 1. Dynamics of vectors arrangement in two-dimensional EEG space of healthy subject 1 according to tests "Eyes open", "Eyes closed", "Plants", "Counting" 
Final Conflquration, dimension 1 vs. dimension 2

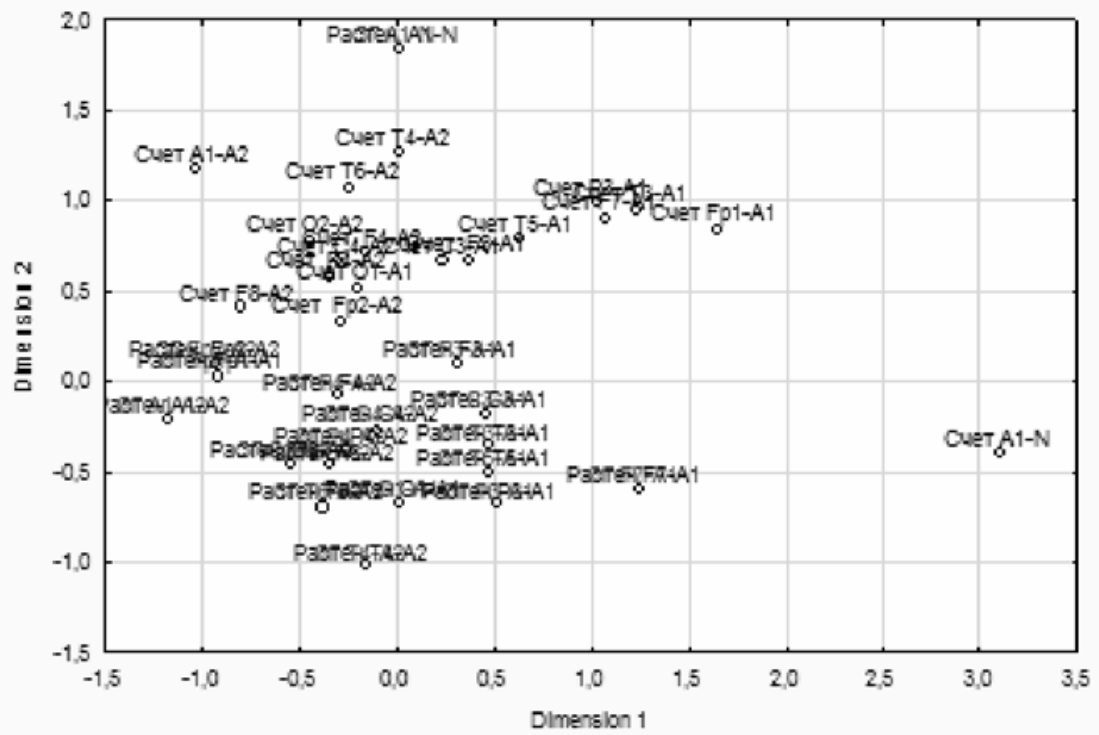

Fig. 2. Dynamics of vectors arrangement in two-dimensional EEG space of healthy subject 2 according to tests "Eyes open", "Eyes closed", "Plants", "Counting"

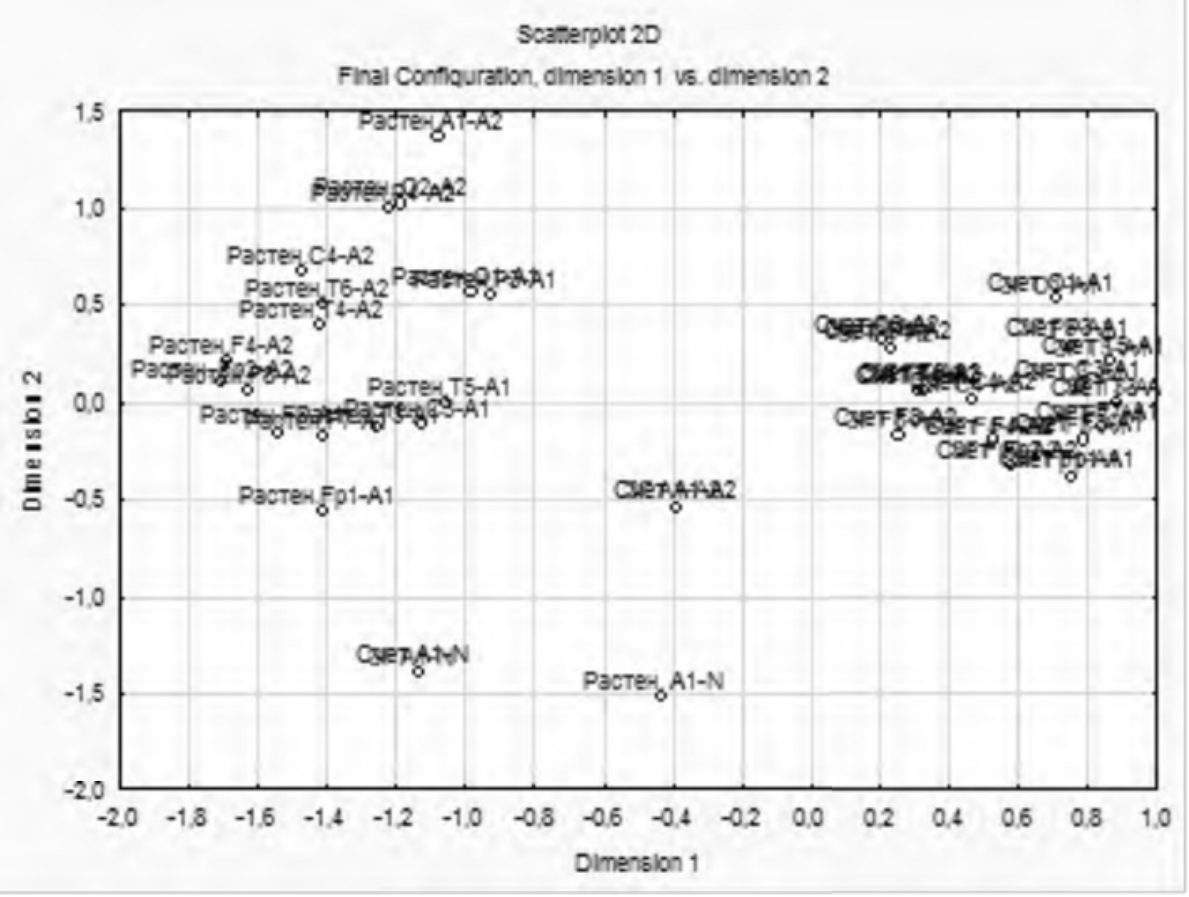

Fig. 3. Dynamics of vectors arrangement in two-dimensional EEG space of healthy subject 3 according to tests "Eyes open", "Eyes closed", "Plants", "Counting" 
In patients with dyscirculatory encephalopathy there is no coincidence of points characterizing spontaneous bioelectric activity of the brain when performing tests "Eyes open", "Eyes closed", "Plants", "Counting".

The application of the method of multidimensional scaling to analyze the similarity and difference of EEG tests "Eyes open", "Eyes closed", "Letter", "Odd one" showed that healthy subjects have a coincidence of spatial coordinates on the tests "Eyes open", "Eyes closed"", "Letter". The formation of a spatial region is also noted with localization of points corresponding to the EEG when performing the "Odd one" test.

In patients, there is no coincidence of spatial coordinates on tests "Eyes open", "Eyes closed" and "Letter". However, the resulting image differs by combining the results into groups of tests. Thus, in the coordinate plane it is possible to select some local areas corresponding to a certain test.

Analysis of data on the tests "Eyes open", "Eyes closed", "Words" showed that again there is almost complete coincidence of the location of points on different tests or grouping in a separate area of the "Words" test results.

Patients have no marked localization, there is a more chaotic distribution of points in the spatial region. In some cases, there is a division into groups of tests.

Comparative analysis of the results of multidimensional EEG scaling of healthy subjects on the tests "Eyes open", "Eyes closed", "Words" again showed almost complete coincidence of spatial coordinates of leads on different tests. In some cases, the selection of a separate area of the "Words" test results is different.

For subjects with dyscirculatory encephalopathy, there is a more chaotic distribution of points on the spatial plane. In some cases, scopes are formed that define one of the tests.

Thus, it can be concluded that for healthy subjects in almost all tests there is a coincidence or close location of spatial coordinates of multidimensional scaling. For patients with dyscirculatory encephalopathy, there is no marked coincidence of coordinates, but in some cases, it is possible to group the data into local areas according to individual tests. The use of the method of multidimensional scaling in the diagnosis of dyscirculatory encephalopathy allows the analysis of the patient's condition, makes it possible to identify the degree of the disease.

In the course of the work, EEG amplitudes of patients were also compared separately for frontal, occipital, parietal, central and temporal leads. As a result, it was found that healthy people are characterized by a vertical arrangement of spatial points corresponding to the same lead in almost all tests.

For patients, the comparison of data on groups of leads showed in some cases the presence of localization of data on different tests and the complete absence of a strictly vertical location of spatial points on a separate lead.

\section{Conclusion}

The aim of the work was to analyze the EEG data by the method of multidimensional scaling to identify differences in the graphs of the dynamics of the location of vectors in coordinate space. The tasks of the work, consisting in the survey of EEG subjects, conducting MS in the system STATISTICA, visual analysis of graphs and drawing conclusions based on the study, are fully fulfilled.

As a result of the study of EEG data, conclusions are drawn:

- $\quad$ when comparing the EEG leads on the tests "Eyes open", "Eyes closed", "Plants", "Counting" in healthy subjects, the coordinates of the MS coincided in almost all tests, in some cases there was a difference between the test "Counting" (or "Plants") and the other tests; for patients with DEP there was no coincidence of points when performing these tests; 
- $\quad$ MS for the analysis of the EEG for tests "Eyes open", "Eyes closed", "Letter", "Odd one" showed that for healthy subjects with the same coordinates on tests "Eyes open", "Eyes closed" "Letter" there was the area of the points of "Odd one" test; the patients had no coincidence of coordinates on these tests, but the results were combined into group;

- $\quad$ analysis on tests "Eyes open", "Eyes closed", "Words" showed almost complete coincidence of points on tests or grouping of test results "Words" of healthy subjects; for patients - more chaotic distribution of points, in some cases the points were divided into groups on tests;

analysis of EEG of healthy subjects on tests "Eyes open", "Eyes closed" showed almost complete coincidence of coordinates on tests, in some cases results of the test "Words" were allocated; for subjects with DEP - more chaotic distribution of points, in some cases the areas defining one of tests were formed;

- comparison of EEG amplitudes by leads revealed: vertical location of points corresponding to one lead for healthy subjects in almost all tests; for patients - localization of test data in some cases and complete absence of vertical location of points on a separate lead.

Similar results were obtained when comparing the remaining tests. The conclusion is made: for healthy subjects in almost all tests there is a coincidence or close location of the coordinates of the MS, for patients with DEP marked coincidence is absent, but in some cases, it is possible to group the data in the area of individual tests.

As a result of the study, it was concluded that MS allows you to classify healthy and sick subjects according to EEG indicators. With this method, in the future it is possible to analyze the patient's health and to identify the degree of the disease. In addition, this study is a classic example of the use of mathematical methods in medicine. Scientists of the Don state technical university have been conducting research in this direction for a long time. $[13,14]$. The results of our research can be used in the process of training bachelors and masters in the field of Biotechnical systems and technologies. In this way, we can implement the recommendations of employers in this area [15]. Graduates of the Department of Instrumentation and biomedical engineering are prepared for scientific research using mathematical methods of analysis.

The testing method presented in this paper is patented by our colleagues [16].

\section{References}

1. N. Sharma, M. H. Kolekar, K. Jha, Y. Kumar, EEG and Cognitive Biomarkers Based Mild Cognitive Impairment Diagnosis, IRBM, 40(2), 113-121 (2019) https://doi.org/10.1016/j.irbm.2018.11.007

2. F. R. Farina, D. D. Emek-Savaş, L. Rueda-Delgado, R. Boyle, H. Kiiski, G. Yener, R. Whelan, A comparison of resting state EEG and structural MRI for classifying Alzheimer's disease and mild cognitive impairment, Neuromage, 21, 116795 (2020) https://doi.org/10.1016/j.neuroimage.2020.116795

3. E. V. Orekhova, M. Elam, V. Yu. Orekhov, Unraveling superimposed EEG rhythms with multi-dimensional decomposition, Journal of Neuroscience Methods, 195(1), 4760 (2011) https://doi.org/10.1016/j.jneumeth.2010.11.010

4. F. Altındiş, B. Yılmaz, S. Borisenok, K. İçöz, Parameter investigation of topological data analysis for EEG signals, Biomedical Signal Processing and Control, 63, 102196 (2021) https://doi.org/10.1016/j.bspc.2020.102196

5. K. Glomb, J. Rué Queralt, D. Pascucci, M. Defferrard, S. Tourbier, M. Carboni, M. Rubega, S. Vulliémoz, G. Plomp, P. Hagmann, Connectome spectral analysis to track 
EEG task dynamics on a subsecond scale, NeuroImage, 221, 117137 (2020) https://doi.org/10.1016/j.neuroimage.2020.117137

6. D. Jenson, D. Thornton, A. W. Harkrider, T. Saltuklaroglu, Influences of cognitive load on sensorimotor contributions to working memory: An EEG investigation of mu rhythm activity during speech discrimination, Neurobiology of Learning and Memory, 166, 107098 (2019) https://doi.org/10.1016/j.nlm.2019.107098

7. P. M. Keune, S. Hansen, T. Sauder, S. Jaruszowic, C. Kehm, J. Keune, E. Weber, M. Schönenberg, P. Oschmann, NeuroImage: Clinical, 22, 101716 (2019) https://doi.org/10.1016/j.nicl.2019.101716

8. P. Marsella, A. Scorpecci, G. Cartocci, S. Giannantonio, A. G. Maglione, I. Venuti, A. Brizi, F. Babiloni, EEG activity as an objective measure of cognitive load during effortful listening: A study on pediatric subjects with bilateral, asymmetric sensorineural hearing loss, International Journal of Pediatric Otorhinolaryngology, 99, 1-7 (2017) https://doi.org/10.1016/j.ijporl.2017.05.006

9. V. Loganina, E. Kuimova, Methods of multi-dimensional statistical analysis for assessing the impact of applying technology of paint formulation: E3S Web Conf. 135, (2019) https://doi.org/10.1051/e3sconf/201913503039

10. C. Oğüt, K. Başar, Assessment of impulsivity with self-report scales and behavioral tasks in symptomatic and remitted patients with major depression: European Neuropsychopharmacology, 27, S807 (2017) https://doi.org/10.1016/S0924977X(17)31462-1

11. B. Jyothia, Y. MadhaveeLathab, P. G. KrishnaMohanc, V. S. K. Reddy, Integrated Multiple Features for Tumor Image Retrieval Using Classifier and Feedback Methods.
Procedia
Computer
Science,
85, $\quad 141-148$
(2016) https://doi.org/10.1016/j.procs.2016.05.200

12. R. Neydorf, V. Krukhmalev, N. Kudinov, V. Pshikhopov, Methods of statistical processing of meteorological data for the tasks of trajectory planning of MAAT feeders: SAE Technical Papers, 7, 100869 (2013) DOI: 10.4271/2013-01-2266.

13. R. Neydorf, A. Neydorf, D. Vučinić, "Cut-Glue" approximation method for strongly nonlinear and multidimensional object dependencies modeling. Advanced Structured Materials, 72, 155-173 (2018) DOI: 10.1007/978-3-319-59590-0_13

14. V. V. Bonilla, C. G. Mosquera, M. D. Mideros, A. V. Litvin, Definition of the elbow movement parameters by means of the analysis of the biceps surface electromiographic signals: Proceedings - 2017 International Conference on Information Systems and Computer Science, INCISCOS 2017, 2017, Pages 141-145 (2018) DOI: 10.1109/INCISCOS.2017.52

15. V. Parshina, E. Kuznetsova, Interaction between industry higher educational institutions and the customer of educational services: E3S Web Conf. 135, (2019) https://doi.org/10.1051/e3sconf/201913504001

16. E. A. Kizhevatova, D. V. Bakuzova, V. P. Omelchenko, V. V. Efremov, Method of diagnosis of cognitive disorders of vascular origin in chronic cerebral ischemia. Pat. 2584651 Russian Federation: IPC A61B5/0476 (2006.01), no. 2015107404/14; declared. 03.03.15; publ. 20.05.16, Byul. 14, 3 (2015) 